\title{
A Note on Hessen berg of Trapezoidal Fuzzy Number Matrices
}

\author{
C.Jaisankar ${ }^{1}$, R. Mani ${ }^{2}$ \\ ${ }^{1,2}$ Department of Mathematics, AVC College (Autonomous)Mannampandal-609305, India.
}

\begin{abstract}
The fuzzy set theory has been applied in many fields such as management, engineering, theory of matrices and so on. in this paper, some elementary operations on proposed trapezoidal fuzzy numbers (TrFNS) are defined. We also have been defined some operations on trapezoidal fuzzy matrices(TrFMs). The notion of Hessenberg fuzzy matrices are introduced and discussed. Some of their relevant properties have also been verified.
\end{abstract}

Keywords: Fuzzy Arithmetic, Fuzzy number, Trapezoidal fuzzy number (TrFN), Trapezoidal fuzzy matrix(TrFM), Hessenberg fuzzy matrix(HFM).

\section{Introduction}

Fuzzy sets have been introduced by Lofti.A.Zadeh[13] Fuzzy set theory permits the gradual assessments of the membership of elements in a set which is described in the interval $[0,1]$. It can be used in a wide range of domains where information is incomplete and imprecise. Interval arithmetic was first suggested by Dwyer [2] in 1951, by means of Zadeh's extension principle [14,15], the usual Arithmetic operations on real numbers can be extended to the ones defined on Fuzzy numbers. Dubosis and Prade [1] has defined any of the fuzzy numbers as a fuzzy subset of the real line [4]. A fuzzy number is a quantity whose values are imprecise, rather than exact as is the case with single - valued numbers.

Trapezoidal fuzzy number's (TrFNs) are frequently used in application. It is well known that the matrix formulation of a mathematical formula gives extra facility to study the problem. Due to the presence of uncertainty in many mathematical formulations in different branches of science and technology.

We introduce trapezoidal fuzzy matrices (TrFMs). To the best of our knowledge, no work is available on TrFMs, through a lot of work on fuzzy matrices is available in literature. A brief review on fuzzy matrices is given below.

Fuzzy matrices were introduced for the first time by Thomason [12] who discussed the convergence of power of fuzzy matrix. Fuzzy matrices play an important role in scientific development. Two new operations and some applications of fuzzy matrices are given in $[, 8,9,10,11]$. Hessenberg matrices play an important role in many application and have been the object of several studies [3,6,7].In recently we proposed the Hessenberg Triangular Fuzzy number matrices[5].

The paper organized as follows, Firstly in section 2, we recall the definition of Trapezoidal fuzzy number and some operations on trapezoidal fuzzy numbers (TrFNs). In section 3, we have reviewed the definition of trapezoidal fuzzy matrix (TrFM) and some operations on Trapezoidal fuzzy matrices (TrFMs). In section 4, we defined the notion of Hessenberg trapezoidal fuzzy matrices. In section 5, we have presented some properties of Hessenberg of trapezoidal fuzzy matrices. Finally in section 6, conclusion is included.

\section{Preliminaries}

In this section, We recapitulate some underlying definitions and basic results of fuzzy numbers.

Definition 2.1 fuzzy set

A fuzzy set is characterized by a membership function mapping the element of a domain, space or universe of discourse $\mathrm{X}$ to the unit interval $[0,1]$. A fuzzy set $\mathrm{A}$ in a universe of discourse $\mathrm{X}$ is defined as the following set of pairs

$\mathrm{A}=\left\{\left(\mathrm{x}, \mu_{\mathrm{A}}(\mathrm{x})\right) ; \mathrm{x} \in \mathrm{X}\right\}$

Here $\mu_{A}: \mathrm{X} \rightarrow[0,1]$ is a mapping called the degree of membership function of the fuzzy set $\mathrm{A}$ and $\mu_{A}(\mathrm{x})$ is called the membership value of $\mathrm{x} \in \mathrm{X}$ in the fuzzy set $\mathrm{A}$. These membership grades are often represented by real numbers ranging from $[0,1]$.

Definition 2.2 Normal fuzzy set

A fuzzy set A of the universe of discourse $\mathrm{X}$ is called a normal fuzzy set implying that there exists at least one $\mathrm{x} \in \mathrm{X}$ such that $\mu_{A}(\mathrm{x})=1$. 
Definition 2.3 Convex fuzzy set

A fuzzy set $\mathrm{A}=\left\{\left(\mathrm{x}, \mu_{A}(\mathrm{x})\right)\right\} \subseteq \mathrm{X}$ is called Convex fuzzy set if all $A_{\propto}$ are Convex set (i.e.,) for every element $x_{1} \in A_{\propto}$ and $x_{2} \in A_{\propto}$ for every $\alpha \in[0,1], \lambda x_{1}+(1-\lambda) x_{2} \in A_{\alpha}$ for all $\lambda \in[0,1]$ otherwise the fuzzy set is called nonconvex fuzzy set.

\section{Definition 2.4 Fuzzy number}

A fuzzy set $\tilde{A}$ defined on the set of real number $\mathrm{R}$ is said to be fuzzy number if its membership function has the following characteristics
i. $\tilde{A}$ is normal
ii. $\tilde{A}$ is convex
iii. The support of $\tilde{A}$ is closed and bounded then $\tilde{A}$ is called fuzzy number.

\section{Definition 2.5 Trapezoidal fuzzy number}

A fuzzy number $\widetilde{A}^{T z L}=\left(a_{1}, a_{2}, a_{3}, a_{4}\right)$ is said to be a trapezoidal fuzzy number if its membership function is given by

$$
\mu_{\bar{\lambda} \pi t}(x)=\left\{\begin{array}{cl}
0 ; x \leq a_{1} \\
\frac{x-a_{1}}{a_{2}-a_{1}} ; a_{1}<x \leq a_{2} \\
1 \quad a_{2} \leq x \leq a_{3} \\
\frac{a_{4}-x}{a_{4}-a_{3}} ; a_{3}<x \leq a_{4} \\
0 \quad ; x \geq a_{4}
\end{array}\right.
$$

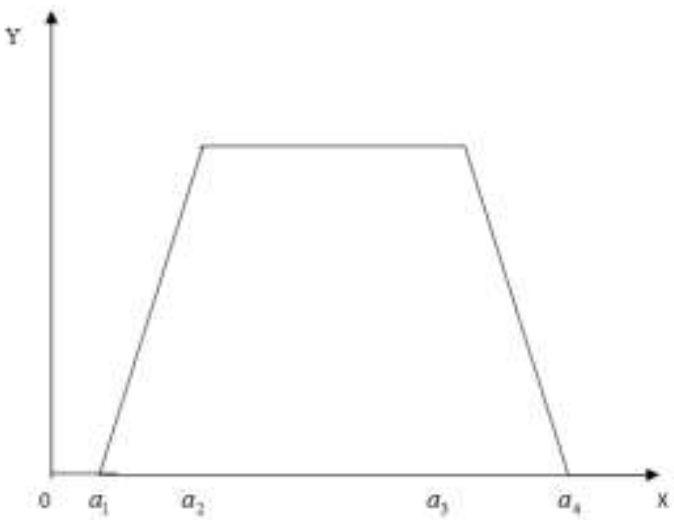

Fig:1 Trapezoidal Fuzzy Number

\section{Definition 2.6 Ranking function}

We defined a ranking function $\mathfrak{R}: \mathrm{F}(\mathrm{R}) \rightarrow \mathrm{R}$ which maps each fuzzy numbers to real line $\mathrm{F}(\mathrm{R})$ represent the set of all trapezoidal fuzzy number. If $\mathrm{R}$ be any linear ranking function

$$
\mathfrak{R}\left(\tilde{A}^{T z L}\right)=\left(\frac{a_{1}+a_{2}+a_{3}+a_{4}}{4}\right)
$$

Also we defined orders on $\mathrm{F}(\mathrm{R})$ by

$\mathfrak{R}\left(\widetilde{A}^{T z L}\right) \geq \mathfrak{R}\left(\tilde{B}^{T z L}\right)$ if and only if $\tilde{A}^{T z L} \geq_{R} \tilde{B}^{T z L}$

$\mathfrak{R}\left(\tilde{A}^{T z L}\right) \leq \mathfrak{R}\left(\widetilde{B}^{T z L}\right)$ if and only if $\tilde{A}^{T z L} \leq_{R} \widetilde{B}^{T z L}$

$\mathfrak{R}\left(\tilde{A}^{T z L}\right)=\mathfrak{R}\left(\widetilde{B}^{T z L}\right)$ if and only if $\tilde{A}^{T z L}={ }_{R} \tilde{B}^{T z L}$ 
Definition 2.7 Arithmetic operations on trapezoidal fuzzy numbers (TrFNs)

Let $\tilde{A}^{T Z L}=\left(a_{1}, a_{2}, a_{3}, a_{4}\right)$ and $\widetilde{B}^{T Z L}=\left(b_{1}, b_{2}, b_{3}, b_{4}\right)$ be trapezoidal fuzzy numbers (TrFNs) then we defined,

\section{Addition}

$\tilde{A}^{T Z L}+\tilde{B}^{T Z L}=\left(a_{1}+b_{1}, a_{2}+b_{2}, a_{3}+b_{3}, a_{4}+b_{4}\right)$

Subtraction

$\tilde{A}^{T Z L}-\widetilde{B}^{T Z L}=\left(a_{1}-b_{4}, a_{2}-b_{3}, a_{3}-b_{2}, a_{4}-b_{1}\right)$

Multiplication

$\widetilde{A}^{T z L} \times \widetilde{B}^{T Z L}=\left(a_{1} \Re(\mathrm{B}), a_{2} \Re(\mathrm{B}), a_{3} \Re(\mathrm{B}), a_{4} \mathfrak{R}(\mathrm{B})\right)$

where $\Re\left(\tilde{B}^{T z L}\right)=\left(\frac{b_{1}+b_{2}+b_{3}+b_{4}}{4}\right) \quad$ or $\Re\left(\tilde{b}^{T z L}\right)=\left(\frac{b_{1}+b_{2}+b_{3}+b_{4}}{4}\right)$

Division

$$
\tilde{A}^{T z L} / \widetilde{B}^{T z L}=\left(\frac{a_{1}}{\mathfrak{R}\left(\widetilde{B}^{T z L}\right)}, \frac{a_{2}}{\mathfrak{R}\left(\widetilde{B}^{T z L}\right)}, \frac{a_{3}}{\mathfrak{R}\left(\widetilde{B}^{T z L}\right)}, \frac{a_{4}}{\mathfrak{R}\left(\widetilde{B}^{T z l}\right)}\right)
$$

Where $\Re\left(\tilde{B}^{T Z L}\right)=\left(\frac{b_{1}+b_{2}+b_{3}+b_{4}}{4}\right)$ or $\Re\left(\tilde{b}^{T Z L}\right)=\left(\frac{b_{1}+b_{2}+b_{3}+b_{4}}{4}\right)$

\section{Scalar multiplication}

$\mathrm{K} \tilde{A}^{T z L}=\left\{\begin{array}{c}\left(k a_{1}, k a_{2}, k a_{3}, k a_{4}\right) \text { if } K \geq 0 \\ \left(k a_{4}, k a_{3}, k a_{2}, k a_{1}\right) \text { if } k<0\end{array}\right.$

\section{Definition 2.8 Zero trapezoidal fuzzy number}

If $\tilde{A}^{T z L}=(0,0,0,0)$ then $\tilde{A}^{T z L}$ is said to be zero trapezoidal fuzzy number. It is defined by 0 .

\section{Definition 2.9 Zero equivalent trapezoidal fuzzy number}

A trapezoidal fuzzy number $\tilde{A}^{T z L}$ is said to be a zero equivalent trapezoidal fuzzy number if $\Re\left(\tilde{A}^{T Z L}\right)=0$. It is defined by $\tilde{0}^{T Z L}$.

\section{Definition 2.10 Unit trapezoidal fuzzy number}

If $\tilde{A}=(1,1,1,1)$ then $\tilde{A}^{T z L}$ is said to be a unit trapezoidal fuzzy number. It is denoted by 1 .

\section{Definition 2.11 Unit equivalent trapezoidal fuzzy number}

A trapezoidal fuzzy number $\widetilde{A}^{T z L}$ is said to be unit equivalent trapezoidal fuzzy number.

If $\Re\left(\tilde{A}^{T Z L}\right)=1$. It is denoted by $\tilde{1}^{T Z L}$.

\section{Definition 2.12 Inverse of trapezoidal fuzzy number}

If $\tilde{a}^{T Z L}$ is trapezoidal fuzzy number and $\tilde{a}^{T Z L} \neq \tilde{0}^{T Z L}$ then we define.

$$
\tilde{a}^{T Z L-1}=\frac{\tilde{1}^{T z l}}{\tilde{a}^{T z L}}
$$

\section{Trapezoidal fuzzy matrices (TRFMS)}

In this section, we introduced the trapezoidal fuzzy matrix and the operations of the matrices some examples provided using the operations.

\section{Definition 3.1 Trapezoidal fuzzy matrix (TrFM)}

A trapezoidal fuzzy matrix of order $\mathrm{m} \times \mathrm{n}$ is defined as $\mathrm{A}=\left(\tilde{a}_{i j}^{T Z L}\right)_{m \times n}$, where $\tilde{a}_{i j}^{T Z L}=\left(a_{i j 1}, a_{i j 2}, a_{i j 3}, a_{i j 4}\right)$ is the $i j^{\text {th }}$ element of A. 


\section{Definition 3.2 Operations on Trapezoidal Fuzzy Matrices (TrFMs)}

As for classical matrices. We define the following operations on trapezoidal fuzzy matrices. Let $\mathrm{A}=\left(\tilde{a}_{i j}^{T Z L}\right)$ and $\mathrm{B}=\left(\tilde{b}_{i j}^{T Z L}\right)$ be two trapezoidal fuzzy matrices (TrFMs) of same order. Then, we have the following

\section{i. Addition}

$$
\mathrm{A}+\mathrm{B}=\left(\tilde{a}_{i j}^{T Z L}+\tilde{b}_{i j}^{T Z L}\right)
$$

\section{ii. Subtraction}

$$
\mathrm{A}-\mathrm{B}=\left(\tilde{a}_{i j}^{T Z L}-\tilde{b}_{i j}^{T Z L}\right)
$$

iii. For $\mathrm{A}=\left(\tilde{a}_{i j}^{T Z L}\right)_{m \times n}$ and $\mathrm{B}=\left(\tilde{b}_{i j}^{T Z L}\right)_{n \times k}$ then $\mathrm{AB}=\left(\tilde{c}_{i j}^{T Z L}\right)_{m \times k}$ where $\tilde{c}_{i j}^{T Z L}=\sum_{p=1}^{n} \tilde{a}_{i p}^{T Z L} \cdot \tilde{b}_{p j}^{T Z L}$, $\mathrm{i}=1,2, \ldots \mathrm{m}$ and $\mathrm{j}=1,2, \ldots \mathrm{k}$

iv. $A^{T}$ or $A^{1}=\left(\tilde{a}_{j i}^{T Z L}\right)$

v. $K A=\left(K \tilde{a}_{i j}^{T Z L}\right)$ where $\mathrm{K}$ is scalar.

\section{Examples}

$$
\begin{aligned}
& \text { 1) If } A=\left[\begin{array}{ll}
(-1,2,3,4) & (2,4,6,8) \\
(-1,1,3,5) & (-2,2,3,5)
\end{array}\right] \text { and } \mathrm{B}=\left[\begin{array}{cc}
(-2,2,3,5) & (-3,3,5,7) \\
(1,4,5,6) & (4,5,9,10)
\end{array}\right] \\
& \text { Then } \mathrm{A}+\mathrm{B}=\left(\tilde{a}_{i j}^{T Z L}+\tilde{b}_{i j}^{T Z L}\right) \\
& A+B=\left[\begin{array}{ll}
(-1,2,3,4) & (2,4,6,8) \\
(-1,1,3,5) & (-2,2,3,5)
\end{array}\right]+\left[\begin{array}{cc}
(-2,2,3,5) & (-3,3,5,7) \\
(1,4,5,6) & (4,5,9,10)
\end{array}\right] \\
& A+B=\left[\begin{array}{ll}
(-3,4,6,9) & (-1,7,11,15) \\
(0,5,8,11) & (2,7,12,15)
\end{array}\right] \\
& \text { 2) If } A=\left[\begin{array}{ll}
(-1,2,3,4) & (2,4,6,8) \\
(-1,1,3,5) & (-2,2,3,5)
\end{array}\right] \text { and } \mathrm{B}=\left[\begin{array}{cc}
(-2,2,3,5) & (-3,3,5,7) \\
(1,4,5,6) & (4,5,9,10)
\end{array}\right] \\
& \text { Then A-B }=\left(\begin{array}{ll}
\tilde{a}_{i j}^{T Z L} & -\tilde{b}_{i j}^{T Z L} \\
&
\end{array}\right) \\
& A-B=\left[\begin{array}{ll}
(-1,2,3,4) & (2,4,6,8) \\
(-1,1,3,5) & (-2,2,3,5)
\end{array}\right]-\left[\begin{array}{cc}
(-2,2,3,5) & (-3,3,5,7) \\
(1,4,5,6) & (4,5,9,10)
\end{array}\right] \\
& A-B=\left[\begin{array}{cc}
(-6,-1,1,2) & (-5,-1,3,11) \\
(-7,-4,-1,4) & (-12,-7,-2,1)
\end{array}\right] \\
& \text { 3) If } A=\left[\begin{array}{ll}
(-1,2,3,4) & (2,4,6,8) \\
(-1,1,3,5) & (-2,2,3,5)
\end{array}\right] \text { and } \mathrm{B}=\left[\begin{array}{cc}
(-2,2,3,5) & (-3,3,5,7) \\
(1,4,5,6) & (4,5,9,10)
\end{array}\right] \\
& \text { Then A.B }=\left(\tilde{a}_{i j}^{T z L} \tilde{b}_{i j}^{T Z L}\right) \\
& \begin{array}{l}
A \cdot B=\left[\begin{array}{ll}
(-1,2,3,4) & (2,4,6,8) \\
(-1,1,3,5) & (-2,2,3,5)
\end{array}\right] \cdot\left[\begin{array}{cc}
(-2,2,3,5) & (-3,3,5,7) \\
(1,4,5,6) & (4,5,9,10)
\end{array}\right] \\
\text { A. } \quad=\left[\begin{array}{cc}
(-1,2,3,4)(2)+(2,4,6,8)(4) & (-1,2,3,4)(4)+(2,4,6,8)(7) \\
(-1,1,3,5)(2)+(-2,2,3,5)(4) & (-1,1,3,5)(4)+(-2,2,3,5)(7)
\end{array}\right] \\
\text { A. B }=\left[\begin{array}{cc}
(6,20,30,40) & (10,36,54,72) \\
(-10,10,18,30) & (-18,18,33,55)
\end{array}\right]
\end{array}
\end{aligned}
$$

\section{Hessen berg Trapezoidal Fuzzy Matrix}

In this section, we introduce the new matrix namely Hessenberg matrix in the fuzzy nature.

\section{Definition 4.1 Lower Hessenberg Fuzzy Matrix}

A Square trapezoidal fuzzy matrix $A=\left(\tilde{a}_{i j}^{T Z L}\right)$ is called an Lower Hessenberg trapezoidal fuzzy matrix if all the entries below the first super diagonal are zero.

i.e. $\tilde{a}_{i j}^{T Z L}=0 ; i+1<j \forall i, j=1,2, \ldots, n$ 


\section{Definition 4.2 Upper Hessenberg Fuzzy Matrix}

A Square trapezoidal fuzzy matrix $A=\left(\tilde{a}_{i j}^{T Z L}\right)$ is called Upper Hessenberg trapezoidal fuzzy matrix if all the entries above the first sub diagonal are zero.

i.e. $\tilde{a}_{i j}^{T Z L}=0 ; i>j+1 \forall i, j=1,2, \ldots, n$

Definition 4.3 Hessenberg Trapezoidal Fuzzy Matrix

A Square trapezoidal fuzzy matrix $A=\left(\tilde{a}_{i j}^{T Z L}\right)$ is called Hessenberg trapezoidal fuzzy matrix (HTrFM). If it is either upper Hessenberg trapezoidal fuzzy matrix and lower Hessenberg trapezoidal fuzzy matrix.

\section{Definition 4.4 Lower Hessenberg Equivalent Trapezoidal Fuzzy Matrix}

A Square trapezoidal fuzzy matrix $A=\left(\tilde{a}_{i j}^{T Z L}\right)$ is called lower Hessenberg equivalent trapezoidal fuzzy matrix if all the entries above the first super diagonal are $\tilde{0}^{T Z L}$

\section{Definition 4.5 Upper Hessenberg Equivalent Trapezoidal Fuzzy Matrix}

A Square trapezoidal fuzzy matrix $A=\left(\tilde{a}_{i j}^{T Z L}\right)$ is called Upper Hessenberg equivalent trapezoidal fuzzy matrix.

If all the entries below the first sub diagonal are $\tilde{0}^{T Z L}$

\section{Definition 4.6 Hessenberg Equivalent Trapezoidal Fuzzy Matrix}

A Square trapezoidal fuzzy matrix $A=\left(\tilde{a}_{i j}^{T Z L}\right)$ is called Hessenberg- equivalent trapezoidal fuzzy matrix. If it is either upper Hessenberg equivalent trapezoidal fuzzy matrix or lower Hessenberg- equivalent trapezoidal fuzzy matrix.

\section{Some Properties of Hessen berg Trapezoidal Matrices}

In this section, we introduced the properties of HTrFM's.

\subsection{Properties of HTrFM (Hessenberg Trapezoidal Fuzzy matrix)}

Property 5.1.1:

The sum of two lower HTrFM's of order $n$ is also a lower HTrFM of order $n$.

Proof:

Let $A=\left(\tilde{a}_{i j}^{T Z L}\right)$ and $B=\left(\tilde{b}_{i j}^{T Z L}\right)$ be two lower HTrFM's

Since A and B are lower HTrFM's then,

$$
\tilde{a}_{i j}^{T Z L}=0 \text { and } \tilde{b}_{i j}^{T Z L}=0 \text {,if } i+1<j \text {, for all } i, j=1, \ldots . n \text {. }
$$

Let $\mathrm{A}+\mathrm{B}=\mathrm{C}$ then $\left(\tilde{a}_{i j}^{T Z L}+\tilde{b}_{i j}^{T Z L}\right)=\left(\tilde{c}_{i j}^{T Z L}\right)$.

Since $i+1<j ; i, j=1, \ldots . n$ then,

$\tilde{c}_{i j}^{T z l}=\tilde{a}_{i j}^{T Z L}+\tilde{b}_{i j}^{T Z L}$

$$
=0
$$

Hence $\mathrm{C}$ is also a lower HTrFM of order $\mathrm{n}$.

\section{Property 5.1.2:}

The sum of two Upper HTrFM's of order $n$ is also an upper HTrFM of order $n$.

Proof:

Let $A=\left(\tilde{a}_{i j}^{T Z L}\right)$ and $B=\left(\tilde{b}_{i j}^{T Z L}\right)$ be two upper HTrFM's

Since A and B are upper HTrFM's.

Then, $\tilde{a}_{i j}^{T Z L}=0$ and $\tilde{b}_{i j}^{T Z L}=0$ for all $i>j+1 ; i, j=1,2, \ldots, n$.

Let $A+B=C$ then $\left(\tilde{a}_{i j}^{T Z L}+\tilde{b}_{i j}^{T Z L}\right)=\left(\tilde{c}_{i j}^{T Z L}\right)$.

Since $i>j+1 ; i, j=1,2, \ldots n$ then

$\tilde{c}_{i j}^{T z l}=\tilde{a}_{i j}^{T Z L}+\tilde{b}_{i j}^{T Z L}$

$$
=0
$$


Hence $\mathrm{c}$ is also an upper HTrFM of order $\mathrm{n}$.

\section{Property 5.1.3:}

The product of lower HTrFM by Constant is also a lower HTrFM.

Proof:

Let $A=\left(\tilde{a}_{i j}^{T Z L}\right)$ be a lower HTFM.

Since A is lower $\mathrm{HTrFM}, \tilde{a}_{i j}^{T Z L}=0, i+1<j$; for all $i, j=1,2, \ldots n$

Let $\mathrm{K}$ be a scalar and $K_{A}=B$, then $\left(K \tilde{a}_{i j}^{T L L}\right)=\left(\tilde{b}_{i j}^{T L L}\right)$.

Since $i+1<j ; i, j=1,2 . . n$ then

$$
\begin{aligned}
\tilde{b}_{i j}^{T Z L} & =K\left(\tilde{a}_{i j}^{T Z L}\right) \\
& =K(0) \\
& =0
\end{aligned}
$$

Hence B is also a lower HTrFM.

\section{Property 5.1.4:}

The Product of an upper HTrFM by a constant is also an upper HTrFM.

Proof:

Let $A=\left(\tilde{a}_{i j}^{T Z L}\right)$ be an upper HTrFM.

Since A is an upper HTrFM $\tilde{a}_{i j}^{T Z L}=0$, For all $i>j+1 ; i, j=1,2 . . n$

Let $\mathrm{K}$ be a scalar and $K A=B$,then $\left(K \tilde{a}_{i j}^{T L L}\right)=\left(\tilde{b}_{i j}^{T Z L}\right)$.

Since $i>j+1 ; i, j=1,2, \ldots, n$ then,

$$
\begin{aligned}
\tilde{b}_{i j}^{T z L} & =K\left(\tilde{a}_{i j}^{T Z L}\right) \\
& =K(0) \\
& =0
\end{aligned}
$$

Hence B is also an upper HTrFM.

\section{Property 5.1.5:}

The Product of two lower HTrFM of order $n$ is also a lower HTrFM of order $n$.

Proof:

Let $A=\left(\tilde{a}_{i j}^{T Z L}\right)$ and $B=\left(\tilde{b}_{i j}^{T Z L}\right)$ be two lower HTrFM's.

Since A and B are lower HTrFM then, $\tilde{a}_{i j}^{T Z L}=0$ and $\tilde{b}_{i j}^{T Z L}=0, i+1<j$; for all $i, j=1,2 \ldots n$

Let $A B=C=\tilde{c}_{i j}^{T z L}$

Where $\tilde{c}_{i j}^{T Z L}=\sum_{\mathrm{k}=1}^{\mathrm{n}} \tilde{a}_{i k}^{T Z L} \cdot \tilde{b}_{k j}^{T Z L}$

We will show that $\tilde{c}_{i j}^{T Z L}=0$,for all $i+1<j ; i, j=1,2 \ldots n$

for $i+1<j$

We have $\tilde{a}_{i k}^{T Z L}=0$ for $k=i+2, i+3 \ldots \ldots \ldots n$ and $\tilde{b}_{k j}^{T Z L}=0$ for $k=1,2, \ldots \ldots i+1$

Therefore $\tilde{c}_{i j}^{T Z L}=\sum_{\mathrm{k}=1}^{\mathrm{n}} \tilde{a}_{i k}^{T Z L} \cdot \tilde{b}_{k j}^{T Z L}$

$$
=\left[\sum_{k=1}^{i+1} \tilde{a}_{i k}^{T Z L} \tilde{b}_{k j}^{T Z L}\right]+\left[\sum_{i+2}^{n} \tilde{a}_{i k}^{T Z L} \cdot \tilde{b}_{k j}^{T Z L}\right]=0
$$

Hence $\mathrm{C}$ is also lower Hessenberg TrFM. 


\section{Property 5.1.6:}

\section{The Product of two upper HTrFMs of order $n$ is also an upper HTrFM of order $n$.}

Proof:

Let $\mathrm{A}=\left(\tilde{a}_{i j}^{T z L}\right)$ and $B=\left(\tilde{b}_{i j}^{T z L}\right)$ be two upper HTrFM.

Since $A$ amd $B$ are upper HTrFMs then, $\tilde{a}_{i j}^{T z L}=0$ and $\tilde{b}_{i j}^{T Z L}=0$, for all $i>j+1 ; i, j=1,2 \ldots n$.

For $i>j+1$ we have $\tilde{a}_{i k}^{T z L}=0$ for $k=1,2, \ldots i$ and

Similarly $\tilde{b}_{k j}^{T z L}=0$ for $k=i+1 \ldots n$.

Therefore $\tilde{c}_{i j}^{T z L}=\sum_{\mathrm{k}=1}^{\mathrm{n}} \tilde{a}_{i k}^{T z L} \cdot \tilde{b}_{k j}^{T z L}$

$$
\begin{aligned}
& =\sum_{k=1}^{i} \tilde{a}_{i k}^{T z L} \cdot \tilde{b}_{k j}^{T z L}+\sum_{k=\mathrm{i}+1}^{n} \tilde{a}_{i k}^{T z L} \cdot \tilde{b}_{k j}^{T z L} \\
& =0
\end{aligned}
$$

Hence $C$ is also upper hessenberg TrFM.

\section{Property 5.1.7:}

The Transpose of an upper HTrFM is a lower HTrFM and vice versa.

Proof:

Let $A=\left(\tilde{a}_{i j}^{T z L}\right)$ be an upper HTrFM.

Since A is an upper HTrFM, $\tilde{a}_{i j}^{T Z L}=0$ for all,$i>j+1 ; i, j=1,2, \ldots, n$

Let B be the transpose of A then $A^{\prime}=B$

i.e. $\left(\tilde{a}_{j i}^{T z L}\right)=\left(\tilde{b}_{i j}^{T z L}\right)$ for all $i>J+1 ; i, j=1,2, \ldots n, \tilde{a}_{j i}^{T z L}=0=\tilde{b}_{i j}^{T z L}$

That is for all $i+1<J ; i, j=1,2, \ldots, n$

$\tilde{b}_{i j}^{T z L}=0$

Hence B is a lower HTrFM.

\section{Conclusion}

In this article, Hessenberg trapezoidal fuzzy matrices are defined and some relevant properties of their Hessenberg fuzzy matrices have also been proved. Few illustrations based on operations of trapezoidal fuzzy matrices have also been justified. In future, these matrices will be apply in the polynomials, generalized fibonacci numbers, and special kind of composition of natural numbers in the domain of fuzzy environment

\section{Reference}

[1]. Dubasis.D and prade.H, (1978), Operations on fuzzy numbers. International journal of systems, vol.9 no.6 pp 613-626

[2]. Dwyer.. (1965), P.S.Fuzzy sets information and control no.8., pp $338-353$

[3]. Edgar Asplud (1959), Inverse of matrices $\left(a_{i j}\right)$ which satisfy $a_{i j}=0$ for $j>i+p$ math S and T, pp 57-60

[4]. Heliporn.S.J(1997), Representation and application of fuzzy numbers, fuzzy sets and systems, vol.91, no.2, pp 259-268.

[5]. Jaisankar.C, Arunvasan.S and Mani.R., On Hessenberg of Triangular fuzzy matrices, ijsret volume 5 issue 12, 586-591.

[6]. Lkebe.Y (1979) on Inverse of Hessenberg Matrices,Linear Algebra Appl.24 pp 93-97

[7]. Romani.F, Rozsa.P, Berwlacquq.R, Favati.P,(1991), on band matrices and their inverses, Linear Algebra, Appl.150 pp 287-296.

[8]. Shyamal.A.K and M.Pal(2004), Two new operators on fuzzy matrices, J.Applied Mathematics and computing 13 pp 91-107.

[9]. Shyamal.A.K and M.Pal(2005), Distance between fuzzy matrices and its applications, Actasiencia Indica, XXXI-M(1) pp 199-204.

[10]. Shyamal.A.K and M.Pal(2005), Distance between fuzzy matrices and its applications-I, J. Natural and physical sciences 19(1) pp 39-58.

[11]. Shyamal.A.K and M.Pal(2007), Trapezoidal fuzzy matrices Iranian journal of fuzzy systems, volume-4 No-1 pp 75-87.

[12]. Thomson M.G,(1977), Convergence of power of the fuzzy matrix , J. Math Anal.Appl., 57 pp 476-480.

[13]. Zadeh.L.A., (1965) Fuzzy sets, Information and control., No.8. pp 338-353.

[14]. Zadeh.L.A., (1978) Fuzzy set as a basis for a theory of possibility, fuzzy sets and systems No-1 pp 3-28.

[15]. Zimmer Mann.H.J.(1996), Fuzzy set theory and its applications, Third Edition, Kluwer Academic Publishers, Boston, Massachusetts. 